\title{
Violencia sexual en hombres y mujeres jóvenes en Chile: Resultados de una encuesta (año 2005) a estudiantes universitarios
}

\author{
Jocelyn A Lehrer ${ }^{1 a}$, Evelyn L Lehrer²b, Pamela B O yarzún ${ }^{3}$. \\ Sexual violence in young men and \\ women in Chile: Results from a 2005 \\ survey of university students
}

Background: Experiences of sexual violence in adolescence and young adulthood have received little attention in Chilean public health research and practice. Aim: To describe the prevalence and contexts of sexual violence victimization in a sample of university students in Chile. Material and Methods: A self-administered, quantitative survey including items on sexual violence was completed by 484 female and 466 male students at a public university in Chile in 2005. Results: Thirty-one percent of women and $21 \%$ of men reported having experienced at least one incident of sexual violence since age 14; the corresponding percentages for the past 12 months were $17 \%$ and $12 \%$, respectively. The perpetrators were identified predominantly as an acquaintance; another important fraction corresponded to a partner or a date. Alcohol or other substances were involved in most cases. Among students who indicated having been assaulted, the incident was reported to the police by none of the men and $2 \%$ of women. Twenty one percent of women and $9 \%$ of men reported having experienced sexual violence before age 14. Conclusions: The high prevalence of sexual violence found in this study indicates that this issue merits further public health attention in Chile (Rev Méd Chile 2009; 137: 599-608).

(Key words: Adolescent; Domestic violence; Sex offences)

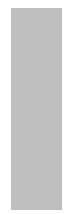

\footnotetext{
Recibido el 10 de diciembre, 2008. Aceptado el 31 de marzo, 2009.

${ }^{1}$ Centro de Estudios de la Prevención del SIDA, Universidad de California, San Francisco.

${ }^{2}$ Departamento de Economía, Universidad de Illinois, Chicago. ${ }^{3}$ Centro de Medicina Reproductiva del Adolescente, Facultad de Medicina, Universidad de Chile.

aDoctor en Ciencias, Salud Pública

${ }^{b}$ Doctor en Economía
}

$\mathrm{E}^{1}$ alto nivel de violencia de género que existe en Chile -física, psicológica y sexual- ha sido analizado en numerosos estudios ${ }^{1-8}$. Hasta ahora el énfasis ha sido en parejas casadas o en uniones

Correspondencia a: Dra. Jocelyn A Lehrer. Center for AIDS Prevention Studies, University of California, San Francisco, CA 94105. Fax: (415) 597-9100. E mail: jlehrer1@gmail.com consensuales. Experiencias de violencia durante la adolescencia o etapas tempranas de la adultez, particularmente en el ámbito de la coerción sexual dentro o fuera del contexto de parejas, han recibido poca atención, tanto en la investigación científica como en programas de prevención y respuesta. Esto representa un vacío importante, estudios en otros países muestran que dicha 
violencia puede tener consecuencias serias, incluyendo un mayor riesgo de problemas psicológicos, sexuales y de salud reproductiva; repercusiones adversas en los estudios y, en el caso de las mujeres, embarazos no deseados ${ }^{9-12}$.

Al igual que en otros países, la evidencia de Estados Unidos de Norteamérica indica que en la población general la prevalencia de agresión sexual contra mujeres es sustancialmente mayor que la de agresión contra hombres ${ }^{13}$. Los estudios basados en muestras de estudiantes universitarios en Estados Unidos de Norteamérica se han enfocado en la violencia dirigida contra mujeres, encontrando una prevalencia de violación de alrededor de 15\% entre los 14/15 años y el día de la encuesta ${ }^{14,15}$. En un estudio nacional conducido en 2000, 2,8\% de las estudiantes reportaron haber experimentado violación o intento de violación en los últimos 7 meses $^{16}$. Con respecto a países en desarrollo, estudios recientes, basados en muestras pequeñas, no representativas, indican elevadas tasas de experiencias sexuales no consentidas en niñas y mujeres jóvenes, y en un grado menor, en niños y hombres jóvenes ${ }^{17}$.

Este estudio es parte de un proyecto más amplio que investiga la violencia de género en una muestra de estudiantes universitarios chilenos. Previamente habíamos publicado un trabajo que analiza la violencia física en mujeres ${ }^{18}$; y otro que es un primer análisis de la violencia sexual, enfocándose en experiencias en mujeres -la prevalencia y los factores que aumentan el riesgo- ${ }^{19}$. El presente estudio utiliza la misma base de datos para presentar un cuadro más completo sobre la violencia sexual en estudiantes chilenos: el enfoque aquí no solamente es en mujeres sino que también en hombres. En forma comparativa, este estudio describe la prevalencia de la violencia sexual en hombres y mujeres y los contextos en que ocurre dicha violencia; también describe la prevalencia de experiencias de violencia sexual durante la niñez.

\section{MATERIAL Y MÉTODO}

Diseño del estudio y encuesta. La "Encuesta de Bienestar Estudiantil, 2005" fue compilada por la primera autora de este trabajo, con el objetivo de recolectar datos cuantitativos sobre la violencia de género en estudiantes universitarios de ambos sexos. El estudio se realizó en una universidad pública en Santiago, con estudiantes inscritos en cursos de Formación General en el semestre de invierno, 2005. La mayoría de las preguntas fueron obtenidas de escalas validadas en Estados Unidos de Norteamérica, adaptadas al contexto chileno, y subsecuentemente revisadas en base a comentarios proporcionados por alumnos de la universidad y también investigadores que han trabajado en el tema de violencia de género. El estudio fue aprobado por el Comité de Ética en la investigación en seres humanos de la universidad.

La encuesta fue conducida en los primeros 25 min de cada clase, y fue autoadministrada, los alumnos contestaron las preguntas marcando sus respuestas en el cuestionario. Había 2.451 estudiantes en las 24 clases de Formación General, con algunos matriculados en más de un curso. Al ser administradas las encuestas, un total de 1.193 estudiantes estuvieron presentes; 970 estudiantes respondieron, un porcentaje de participación de $81 \%$. Algunos de los cuestionarios entregados sin contestar correspondían a estudiantes que ya habían completado la encuesta en otro curso. La muestra que se obtuvo abarcó estudiantes en todos los programas educacionales ofrecidos por la universidad ( $\mathrm{n}=484$ mujeres, 466 hombres). Más detalles sobre la metodología están disponibles en otras publicaciones ${ }^{18,19}$.

Variables. La encuesta incluyó cinco preguntas sobre diferentes formas de violencia sexual, basadas en escalas desarrolladas por Koss y colegas $^{20}$ y por Straus y colegas ${ }^{21}$. Las preguntas sobre los contextos de la violencia están basadas en parte en las utilizadas por Abbey y colegas ${ }^{22}$. Las tablas discutidas más abajo describen las variables.

Estadística. El programa SAS, versión 9.1 (SAS Institute, Cary, NC) fue utilizado para el análisis de los datos. Se generaron frecuencias para los incidentes de violencia sexual desde los 14 años y en los últimos 12 meses, y se hicieron pruebas para determinar si existían diferencias estadísticamente significativas entre mujeres y hombres en las prevalencias de reportar algún tipo de violencia sexual en cada marco de tiempo. Luego se generaron frecuencias para describir los contextos del incidente de violencia sexual desde los 14 años considerado como el más severo por el sujeto. 
Finalmente, se generaron frecuencias para describir las experiencias de violencia sexual antes de los 14 años, y el sexo de los perpetradores de violencia sexual antes o después de los 14 años.

En la serie de 5 preguntas sobre violencia sexual, casos con más de una pregunta sin respuesta fueron eliminados. (En la muestra femenina se eliminaron 51 y 26 casos en los análisis sobre violencia en los últimos 12 meses y desde los 14 años, respectivamente; las cifras correspondientes en la muestra masculina fueron 61 y 41 casos). Si un sujeto contestó todas las preguntas menos una, se asumió que el tipo de violencia que fue dejado en blanco no ocurrió. Esta imputación, usando la categoría modal -que no hubo experiencia de violencia sexual- genera estima- ciones conservadoras de prevalencia. Para los otros análisis, se procedió con un conjunto de variables a la vez, eliminando los casos sin respuestas para ese conjunto, minimizando de este modo la pérdida de datos.

Algunas tablas utilizan la muestra completa; otras están basadas en subgrupos. La columna correspondiente señala el número que quedó después de eliminar observaciones que carecían de respuestas para el tema analizado en esa tabla.

\section{RESULTADOS}

La Tabla 1 muestra las prevalencias de diversas formas de violencia sexual desde los 14 años y en

Tabla 1. Experiencias de violencia sexual antes de los 14 años y en los últimos 12 mesesa,b (\%)

\begin{tabular}{|c|c|c|c|c|}
\hline & \multicolumn{2}{|c|}{ Mujeres } & \multicolumn{2}{|c|}{ H ombres } \\
\hline & $\begin{array}{l}\text { Panel A: } \\
\text { Últimos } \\
12 \text { meses } \\
n=433\end{array}$ & $\begin{array}{r}\text { Panel B: } \\
\text { Desde los } \\
14 \text { años } \\
n=458\end{array}$ & $\begin{array}{l}\text { Panel A: } \\
\text { Últimos } \\
12 \text { meses } \\
n=405\end{array}$ & $\begin{array}{r}\text { Panel B: } \\
\text { Desde los } \\
14 \text { años } \\
n=425\end{array}$ \\
\hline \multicolumn{5}{|l|}{ Violación } \\
\hline Alguien me forzó a tener sexo usando fuerza física & 0,9 & 2,0 & 0,0 & 0,2 \\
\hline $\begin{array}{l}\text { Alguien me forzó a tener sexo usando amenazas u } \\
\text { otras presiones verbales }\end{array}$ & 1,2 & 3,5 & 0,7 & 2,4 \\
\hline $\begin{array}{l}\text { Alguien tuvo sexo conmigo cuando yo había estado } \\
\text { tomando alcohol y/o usando drogas, y yo no estaba en } \\
\text { condiciones de parar lo que estaba ocurriendo. }\end{array}$ & 4,2 & 6,8 & 5,2 & 8,7 \\
\hline $\begin{array}{l}\text { Algún tipo de violación: Sujeto respondió } \\
\text { afirmativamente a por lo menos una de las tres } \\
\text { preguntas más arriba }\end{array}$ & 5,5 & 9,4 & 5,4 & 10,1 \\
\hline $\begin{array}{l}\text { Intento de Violación } \\
\text { Alguien trató de tener sexo conmigo usando } \\
\text { amenazas, argumentos, o fuerza física, pero esto no } \\
\text { sucedió. }\end{array}$ & 4,2 & 10,7 & 2,0 & 4,2 \\
\hline $\begin{array}{l}\text { Otras formas de violencia sexual } \\
\text { Sujeto indica haber tenido alguna otra experiencia } \\
\text { sexual no deseada, como besos forzados o manoseos }\end{array}$ & 12,7 & 25,1 & 6,7 & 13,4 \\
\hline Variable de resumen: Alguna violencia sexual ${ }^{\mathrm{C}}$ & 17,1 & 31,2 & 11,6 & 20,5 \\
\hline
\end{tabular}

aMuestra incluye todos los sujetos: 484 mujeres; 466 hombres.

${ }^{b}$ Categorías no son mutualmente exclusivas, sujetos que indicaron más de un tipo de violencia sexual contribuyen a los porcentajes de cada fila.

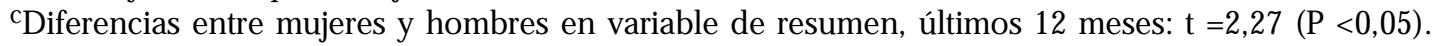

Diferencias entre mujeres y hombres en variable de resumen, desde los 14 años: $t=3,68(P<0,01)$. 
los últimos 12 meses. Cabe notar que en la encuesta, la palabra "sexo" fue definida como refiriéndose a sexo vaginal, oral 0 anal. Los resultados señalan que $9,4 \%$ de las mujeres y $10,1 \%$ de los hombres reportaron haber sido víctimas de violación desde los 14 años; los porcentajes correspondientes a los últimos 12 meses son 5,5\% y 5,4\%, respectivamente. En la muestra femenina, $55,4 \%$ de los casos de violación desde los 14 años ocurrieron cuando la joven había estado tomando alcohol o usando drogas, y no estaba en condiciones de detener lo que estaba ocurriendo; el porcentaje correspondiente en la muestra masculina fue $77,1 \%$. Intentos de violación desde los 14 años fueron reportados por $10,7 \%$ de las mujeres y $4,2 \%$ de los hombres. La variable de resumen -algún tipo de violencia sexual- muestra que $31,2 \%$ de las mujeres y $20,5 \%$ de los hombres reportaron algún incidente desde los 14 años; las cifras correspondientes para los últimos 12 meses fueron 17,1\% y 11,6\%, respectivamente; ambas diferencias entre mujeres y hombres son estadísticamente significativas.

Las Tablas 2 a 6 muestran información sobre los contextos de la experiencia sexual no consentida más severa desde los 14 años. Estas preguntas fueron respondidas por aproximadamente $75 \%$ a $80 \%$ de los sujetos que indicaron haber experimentado violencia sexual. Los resultados muestran que $37,3 \%$ de las mujeres y $50,7 \%$ de los hombres identificaron al agresor como un conocido ("amigo (a), otro estudiante, alguien conocido"). Aproximadamente $13 \%$ de las mujeres y hombres señalaron que fue "alguien con quien estaba en una cita;" $26,4 \%$ de las mujeres y $20,9 \%$ de los hombres indicaron que fue un pololo (a), ex-pololo (a) o pareja sexual (Tabla 2).

En $57,0 \%$ de los incidentes más severos en la muestra femenina y $63,8 \%$ de aquellos en la muestra masculina, la víctima, agresor o ambos habían consumido alcohol u otras substancias (Tabla 3). Los lugares más frecuentes de asalto fueron la casa de la víctima o el agresor, y casas donde había una fiesta (Tabla 4).

En respuesta a la pregunta sobre si le contaron a alguien acerca del incidente, $25,0 \%$ de las mujeres y $25,7 \%$ de los hombres dieron una respuesta negativa. Entre aquellos que le contaron a alguien, más de $80 \%$ de los sujetos de ambos sexos lo hicieron a un amigo (a). Ningún hombre y $2,5 \%$ de las mujeres indicaron que lo reportaron a la policía (Tabla 5). La mitad de los hombres y $34,6 \%$ de las mujeres que experimentaron violación o intento de violación y no lo reportaron a la policía indicaron no haberlo hecho en parte porque pensaron que lo que sucedió no fue suficientemente serio o un crimen. Otras razones indicadas frecuentemente fueron no haber estado segura (o) que la intención del agresor (a) fue de lastimar $(26,9 \%$ de las mujeres, $14,3 \%$ de los hombres) y haber estado avergonzado (a) (19,2\% de las mujeres, $14,3 \%$ de los hombres) (Tabla 6).

Finalmente, $20,7 \%$ de las mujeres y $9,2 \%$ de los hombres reportaron haber tenido alguna experiencia de violencia sexual antes de los 14 años; la diferencia es significativa (Tabla 7). Los agresores fueron identificados como miembros de la familia por $46,5 \%$ de las mujeres agredidas y por $28,0 \%$ de los hombres agredidos; $24,0 \%$ de los hombres respondieron que el agresor fue "otro adulto"

Tabla 2. Agresor en incidente más severo de contacto sexual no consentido desde los 14 años (\%)

\begin{tabular}{|lrr|}
\hline & $\begin{array}{c}\text { M ujeres } \\
\mathbf{n = 1 1 0}\end{array}$ & $\begin{array}{r}\text { H ombres } \\
\mathbf{n}=67\end{array}$ \\
\hline Alguien con quien estaba en una cita & 12,7 & 13,4 \\
Pololo(a) / ex-pololo(a) o pareja sexual & 26,4 & 20,9 \\
Amigo(a), otro estudiante, alguien conocido & 37,3 & 50,7 \\
Profesor(a) & 0,0 & 1,5 \\
Un extraño & 16,4 & 7,5 \\
Miembro de la familia & 7,3 & 6,0 \\
\hline
\end{tabular}

aSubgrupo: 143 mujeres y 87 hombres que indicaron algún contacto sexual no consentido desde los 14 años. 
Tabla 3. Consumo de alcohol u otras sustancias durante incidente más severo de contacto sexual no consentido desde los 14 años ${ }^{\mathrm{a}}$

\begin{tabular}{|lcc|}
\hline & $\begin{array}{c}\text { Mujeres } \\
\mathbf{n = 1 0 7}\end{array}$ & $\begin{array}{r}\text { Hombres } \\
\mathbf{n}=69\end{array}$ \\
\hline $\begin{array}{l}\text { Sólo víctima consumió alcohol u } \\
\text { otras sustancias }\end{array}$ & 3,7 & 8,7 \\
$\begin{array}{l}\text { Sólo perpetrador consumió alcohol } \\
\text { u otras sustancias } \\
\begin{array}{l}\text { Ambos consumieron alcohol u } \\
\text { otras sustancias }\end{array}\end{array}$ & 13,1 & 11,6 \\
$\begin{array}{l}\text { Variable de resumen: Alcohol u } \\
\text { otras substancias involucrados } \\
\text { (perpetrador, víctima, o ambos) }\end{array}$ & 40,2 & 43,5 \\
\hline
\end{tabular}

aSubgrupo: 143 mujeres y 87 hombres que indicaron algún contacto sexual no consentido desde los 14 años.

Tabla 4. Lugar donde ocurrió el incidente más severo de contacto sexual no consentido desde los 14 añosa

\begin{tabular}{|lrr|}
\hline & $\begin{array}{r}\text { Mujeres } \\
\mathbf{n = 1 0 9}\end{array}$ & $\begin{array}{r}\text { Hombres } \\
\mathbf{n = 6 7}\end{array}$ \\
\hline Fiesta en una casa & 30,5 & 38,8 \\
Fiesta en una discoteca & 9,2 & 10,5 \\
Casa de un estudiante & 2,8 & 4,5 \\
Casa de víctima o agresor & 38,5 & 29,9 \\
Otro lugar & 19,3 & 16,4 \\
\hline
\end{tabular}

aSubgrupo: 143 mujeres y 87 hombres que indicaron algún contacto sexual no consentido desde los 14 años.

(Tabla 8). Con respecto al sexo de los agresores de violencia sexual a lo largo de la vida (antes o después de los 14 años), 91,4\% de las mujeres agredidas reportaron que los perpetradores fueron únicamente hombres; $68,0 \%$ de los hombres agredidos indicaron que los perpetradores fueron solamente mujeres (Tabla 9).

\section{DisCUSIÓN}

En esta encuesta, contestada por 950 estudiantes en una universidad pública chilena, se encontraron altas tasas de experiencias de violencia sexual en mujeres y hombres. Las mujeres reportaron una prevalencia de victimización significativamen- te mayor que los hombres: $31,2 \%$ comparado con 20,5\% desde los 14 años, y 17,1\% comparado con $11,6 \%$ en los últimos 12 meses.

Los resultados de la muestra femenina muestran una prevalencia de violación desde los 14 años de 9,4\%, menor que la prevalencia de $15 \%$ que se ha encontrado para alumnas universitarias en Estados Unidos de Norteamérica en un marco similar de tiempo, usando definiciones similares de violación ${ }^{14,15}$. Cabe notar que la muestra en este estudio estaba compuesta en gran parte de estudiantes en etapas tempranas de sus estudios universitarios, en contraste a las muestras norteamericanas. Además, la tendencia a reportar incidentes de violencia sexual podría ser relativamente baja en un país tradicional como 
Tabla 5. C on respecto al incidente más severo de contacto sexual no consentido desde los 14 años: ¿Le contó el sujeto a alguien?a,b (\%)

\begin{tabular}{|lrr|}
\hline & $\begin{array}{r}\text { Mujeres } \\
\mathbf{n = 1 0 8}\end{array}$ & $\begin{array}{r}\text { H ombres } \\
\mathbf{n}=\mathbf{7 0}\end{array}$ \\
\hline Panel A & & \\
$\quad$ No le contó a nadie & 25,0 & 25,7 \\
Panel B & & \\
Le contó a: & & 5,8 \\
Madre/madrastra & 18,5 & 3,9 \\
Padre/padrastro & 7,4 & 5,8 \\
Hermano/hermana & 9,9 & 1,9 \\
Otros familiares & 13,6 & 88,5 \\
Amigo (a) & 84,0 & 3,9 \\
Psicólogo/visitadora social & 11,1 & 0,0 \\
Médico & 2,5 & 1,9 \\
Pastor, sacerdote u otro líder religioso & 0,0 & 0,0 \\
Profesor(a) & 1,2 & 0,0 \\
Policía & 2,5 & 7,7 \\
Otro & 14,8 & \\
\hline
\end{tabular}

${ }^{a}$ Categorías en Panel B no son mutualmente exclusivas.

bSubgrupo, Panel A: 143 mujeres y 87 hombres que indicaron algún contacto sexual no consentido desde los 14 años.

Subgrupo, Panel B: 81 mujeres y 52 hombres que indicaron algún contacto sexual no consentido desde los 14 años y haberle contado a alguien.

Tabla 6. Sujetos que experimentaron violación o intento de violación desde los 14 años y no lo reportaron a la policía: razones por no reportara,b (\%)

\begin{tabular}{|lrr|}
\hline & $\begin{array}{r}\text { M ujeres } \\
\mathbf{n = 5 2}\end{array}$ & $\begin{array}{r}\text { Hombres } \\
\mathbf{n = 4 2}\end{array}$ \\
\hline No pensé que lo que me sucedió fuera suficientemente serio, & 34,6 & 50,0 \\
o un crimen & 1,9 & 0,0 \\
No supe cómo ir a la policía & 9,6 & 7,1 \\
Si le contaba a la policía ellos no iban a reaccionar & 7,7 & 2,4 \\
Pensé que no me creerían & 11,5 & 4,8 \\
Tenía miedo de no ser bien tratado(a) por el sistema judicial & 26,9 & 14,3 \\
No estaba seguro(a) de que la persona que hizo esto & & \\
realmente quizo lastimarme & 1,9 & 4,8 \\
No quise que mi relación con esa persona terminara & 3,9 & 9,5 \\
Miedo de represalias por la persona que lo hizo & 19,2 & 14,3 \\
Me sentí avergonzado(a) & 34,6 & 26,2 \\
Otra razón & & \\
\hline
\end{tabular}

${ }^{a}$ Categorías no son mutualmente exclusivas.

bubgrupo: 69 mujeres y 50 hombres que indicaron alguna experiencia de violación o intento de violación desde los 14 años y no la reportaron a la policía. 
Tabla 7. Experiencias de violencia sexual antes de los 14 años $5^{a, b}(\%)$

\begin{tabular}{|lrr|}
\hline & $\begin{array}{r}\text { M ujeres } \\
\mathbf{n = 4 6 5}\end{array}$ & $\begin{array}{r}\text { H ombres } \\
\mathbf{n}=\mathbf{4 3 7}\end{array}$ \\
\hline Sujeto reporta que antes de los 14 años: & & \\
Hubo alguna persona que le hizo tener sexo contra su voluntad & 1,9 & 2,1 \\
Experimentó alguna otra forma de experiencia sexual no deseada & 20,4 & 8,7 \\
Variable de resumen: Alguna experiencia de violencia sexualc & 20,7 & 9,2 \\
\hline
\end{tabular}

aMuestra incluye todos los sujetos: 484 mujeres; 466 hombres.

${ }^{b}$ Categorías no son mutualmente exclusivas.

'Diferencia entre mujeres y hombres en promedio de variable de resumen: $t=4,93$ ( $P<0,01)$.

Tabla 8. Agresor en experiencia de violencia sexual más severa antes de los 14 añosa $(\%)$

\begin{tabular}{|lrr|}
\hline & $\begin{array}{r}\text { Mujeres } \\
\mathbf{n = 7 1}\end{array}$ & $\begin{array}{r}\text { H ombres } \\
\mathbf{n}=\mathbf{2 5}\end{array}$ \\
\hline Pololo (a) o pareja sexual & 5,6 & 16,0 \\
Amigo (a) u otro estudiante & 11,3 & 16,0 \\
Miembro de la familia & 46,5 & 28,0 \\
Pareja de miembro de la familia & 7,0 & 4,0 \\
Profesor (a) & 1,4 & 4,0 \\
Extraño & 9,9 & 4,0 \\
Otro adulto & 18,3 & 24,0 \\
No recuerda & 0,0 & 4,0 \\
\hline
\end{tabular}

aSubgrupo: 96 mujeres y 40 hombres que indicaron algún contacto sexual no consentido antes de los 14 años.

Tabla 9. Sexo de agresores en actos de violencia sexual, antes o después de los 14 años ${ }^{a}(\%)$

\begin{tabular}{|lrr|}
\hline & $\begin{array}{r}\text { Mujeres } \\
\mathbf{n = 1 3 9}\end{array}$ & $\begin{array}{r}\text { Hombres } \\
\mathbf{n}=\mathbf{7 8}\end{array}$ \\
\hline Mujeres solamente & 2,2 & 68,0 \\
Mujeres y hombres & 6,5 & 11,5 \\
Hombres solamente & 91,4 & 20,5 \\
\hline
\end{tabular}

aSubgrupo: 177 mujeres y 108 hombres que indicaron algún contacto sexual no consentido, antes 0 después de los 14 años.

Chile, donde hay un nivel mínimo de discurso social sobre este tema ${ }^{11}$.

El presente estudio revela una alta tasa de violación en la muestra masculina; la tasa verdadera probablemente es aún más elevada por la misma razón notada más arriba. Este hallazgo merece más atención en investigaciones futuras, junto con aquél que muestra que en una proporción importante de la violencia sexual experimentada por hombres, el agresor es una persona del 
mismo sexo. Consistente con estos resultados, un estudio reciente en Lima, Perú, encuentra que entre hombres que reportan alguna experiencia heterosexual, 11\% indican coerción la primera vez que tuvieron sexo; entre aquellos que reportan alguna experiencia homosexual, $45 \%$ indican una iniciación no consentida ${ }^{23}$.

En este estudio, los amigos (as) fueron los confidentes más comunes cuando los estudiantes le contaron a alguien sobre sus experiencias de violencia sexual, indicando la importancia de establecer programas para educar a los pares sobre cómo identificar señales de riesgo en sus amistades; proveerles apoyo con sensibilidad; y ayudarlos a reducir su riesgo y a encontrar más ayuda cuando fuera necesario.

El hallazgo que ningún hombre, y solamente $2 \%$ de las mujeres, reportaron el incidente más severo de violencia sexual desde los 14 años a la policía apoya la noción que las estadísticas basadas en reportes oficiales subestiman seriamente la magnitud del problema. Una tasa importante de mujeres y hombres que experimentaron violación o intento de violación dijeron no haberlo reportado a la policía porque estimaron que lo sucedido no fue lo suficientemente serio o un crimen, o por no haber estado segura (o) que la intención de la persona que perpetró la coerción sexual fue de lastimar. Estos resultados sugieren la importancia de aumentar el esfuerzo dirigido a educar a la juventud sobre las definiciones de violación, asalto sexual y consentimiento, los derechos básicos de cada persona en el contexto de la sexualidad y las protecciones de la ley.

El hallazgo que consumo de alcohol u otras substancias por la víctima o perpetrador estaba involucrado en la mayoría de los incidentes de violencia sexual más severos es consistente con resultados en poblaciones universitarias en Estados Unidos de Norteamérica $^{22}$ y también es relevante para el diseño de programas de prevención. Una reciente revisión crítica de la literatura de Estados Unidos de Norteamérica sobre asalto sexual y consumo de alcohol enfatiza la importancia de integrar elementos de prevención de consumo de alcohol a programas de prevención de violencia sexual (orientados hacia posibles agresores) y a programas de disminución de riesgo (orientados hacia posibles víctimas) ${ }^{24}$.
Los resultados que muestran que los asaltos sexuales más severos desde los 14 años fueron perpetrados mayormente por un conocido, y que otro número importante ocurrió en el contexto de relaciones de pareja o en una cita, también son significativos. En Estados Unidos de Norteamérica comúnmente se utilizan dos términos, reflejando correspondientes realidades acerca de la violación, "acquaintance rape", que se puede traducir como "violación por un conocido", y "date rape", que se puede traducir como "violación en una cita". "Acquaintance rape" ha sido definido como "sexo no consentido entre individuos que se conocían antes del acto sexual" y "date rape" como "un subconjunto de 'acquaintance rape' en que el sexo no consentido ocurre entre dos personas que están en una relación romántica"25.

La importancia del lenguaje para reflejar y nombrar realidades sociales o culturales es ampliamente reconocida ${ }^{26}$. Los resultados del presente estudio sugieren que los fenómenos de "violación en una cita" y "violación por un conocido" existen como problemática en la población de estudiantes universitarios chilenos. Dada esta aparente realidad, sería útil que la Salud Pública en Chile adopte términos -éstos u otros semejantes- para nombrar estos problemas formalmente e identificarlos como temas merecedores de más estudio y esfuerzos de prevención y respuesta.

Finalmente, el abuso sexual en menores puede tener consecuencias graves para su bienestar físico, mental y social, a corto y largo plazo, y es un factor que aumenta el riesgo de re-victimización más adelante, durante la adolescencia y temprana adultez ${ }^{27,28}$. Las altas tasas de violencia sexual que este estudio encuentra en niñas y niños subrayan la importancia de prestarle atención en Chile a este serio problema de salud pública.

El presente estudio tiene algunas limitaciones. Aunque la muestra abarcó estudiantes en los diversos programas educacionales de la universidad, los resultados no pueden generalizarse a todo el cuerpo estudiantil. Los resultados probablemente subestiman las prevalencias, ya que las víctimas de violencia de género frecuentemente no reportan lo ocurrido 29,30 , una tendencia que probablemente es pronunciada en la sociedad 
conservadora de Chile. Además, un número considerable de estudiantes estuvieron ausentes al ser administradas las encuestas, probablemente incluyendo una proporción importante de individuos con mayor riesgo. En investigaciones futuras sería deseable usar categorías más detalladas con respecto a los agresores en incidentes de violencia sexual.

En conclusión, este estudio descriptivo sobre la violencia sexual en estudiantes universitarios chilenos demuestra altas prevalencias en mujeres y hombres. Los resultados indican que sería importante recolectar más datos cuantitativos y cualitativos con muestras representativas de estudiantes de ambos sexos y con otros sectores de la población joven. Si una masa crítica de estudios confirma la presencia de una problemática de violencia sexual en la juventud chilena, será claramente necesario empezar a desarrollar, implementar y evaluar programas para prevenir y responder a esta preocupación de salud pública.

\section{REFERENCIAS}

1. LaRRAín SH. Violencia Puertas Adentro: La Mujer Golpeada. Santiago, Chile: Editorial Universitaria; 1994.

2. Mc WhiRTer PT. La violencia privada: domestic violence in Chile. Am Psychol 1999; 54: 37-40.

3. Bacigalupe G. Family violence in Chile: Political and legal dimensions in a period of demographic transition. Violence Against Women 2000; 6: 427-48.

4. SERNAM. Detección y análisis de la la prevalencia de la violencia intrafamiliar. Santiago, Chile; 2002.

5. Aluaga P, Ahumada S, Marfull M. Violencia contra las mujeres: Un problema de todos. Rev Chil Obstet Ginecol 2003; 68: 75-8.

6. Cebalo R, Ramírez C, Castilo M, Cabalero A, Lozofy B. Domestic violence and women's mental health in Chile. Psychology Women Quartely 2004; 28: 298-308.

7. Hassan F, Sadowski LS, Bangdiwala SI, VizcarRa B, Ramiro L, De Paula CS et al. Physical intimate partner violence in Chile, Egypt, India and the Philippines. Inj Control Saf Promot 2004; 11: 111-6.

8. Donoso SE. Violencia Contra Mujeres en Chile: Un Problema de Salud Pública. Rev Chil Obstet Ginecol 2007; 72 Disponible en http://www.scielo.cl/ scielo.php?script $=$ sci arttext\&pid $=$ S0717 75262007000500001 (consultado el 1 diciembre, 2008).

9. Rickert VI, Vaughan RD, Wiemann CM. Adolescent dating violence and date rape. Current Opinion in Obstetrics and Gynecology 2002; 14: 495-500.
Los resultados también sugieren la importancia de iniciar investigaciones paralelas en otros países latinoamericanos donde hay una carencia similar de datos y programas en esta área.

\section{Agradecimientos}

Este estudio es parte de un proyecto más amplio sobre violencia de género en Chile. Le agradecemos a los administradores de la universidad donde fue conducida la encuesta por su entusiástica cooperación en este proyecto y por facilitar nuestro acceso a todos los cursos de Formación General ofrecidos en el semestre de invierno, 2005. Vaughn Rickert, Psy.D. proporcionó valiosas sugerencias con respecto al cuestionario y diseño de la encuesta. También recibimos comentarios útiles de dos revisores anónimos de Revista Médica Chilena y de participantes en seminarios presentados en la Universidad de Chile, Escuela de Medicina y Departamento de Ciencias Sociales (Agosto, 2006).

10. Rickert VI, VAughan RD, Wiemann CM. Violence against young women: implications for clinicians. Contemporary OB/GYN 2003; 48: 30-45.

11. Krug EG, Mercy JA, Dahlberg LL, Zwi A. World Report on Violence and Health, Geneva: WHO; 2002.

12. Krug EG, Mercy JA, Dahlberg LL, Zwi A. Beyond victims and villains: addressing sexual violence in the education sector, Panos Report, London: Panos Institute; 2003, $\mathrm{N}^{0} 47$.

13. Tjaden P, Thoennes NN. Prevalence, incidence, and consequences of violence against women: Findings from the National Violence Against Women Survey. National Institutes of Justice Centers for Disease Control and Prevention, Research Brief; 1998.

14. Koss MP, Gidycz CA, Wiesniewski N. The scope of rape: Incidence and prevalence of sexual aggression and victimization in a national sample of higher education students. J Counsel Clin Psych 1987; 55: 162-70.

15. Brener ND, Mcmahon PM, WarRen CW, Douglas KA. Forced sexual intercourse and associated health-risk behaviors among female college students in the United States. J Consult Clin Psychol 1999; 67: 252-9.

16. Fisher BS, CuLen FT, TuRnER MG. The Sexual Victimization of College Women. U.S. Department of Justice, Office of Justice Programs, 2000. Disponible en http://www.ncjrs.gov/pdffiles1/nij/182369.pdf. Consultado el 14 de noviembre, 2008.

17. Jejeebhoy SJ, Shah I, Thapa S. (Editores) Sex Without Consent: Young People in Developing Countries. London: Zed Books; 2005. 
18. Lehrer JA, Lehrer VL, Lehrer EL, ZhenXiang Z. Physical dating violence among college students in Chile. IZA Discussion Paper \# 2753, 2007. Disponible en ERLINK "http://www.iza.org/" Consultado el 18 de octubre, 2008.

19. Lehrer JA, Lehrer VL, Lehrer EL, Oyarzún PB. Prevalence of and risk factors for sexual victimization in college women in Chile. Int Fam Plann Persp 2007; 33: 168-75.

20. Koss MP, Abbey A, Campbell R, Cook S, Norris J, Testa $\mathrm{M}$ ET AL. The Sexual Experiences Survey Short Form Victimization, Tucson, AZ, USA: University of Arizona; 2006.

21. Straus MA, Hamby SL, Warren WL. The Conflict Tactics Scales Handbook: Revised Conflict Tactics Scale (CTS2) and CTS: Parent-Child Version (CTSPC), Los Angeles, CA, USA: Western Psychological Services; 2003.

22. Aвbey A, Thomson Ross L, Mcduffie D, Mcauslan P. Alcohol and dating risk factors for sexual assault among college women. Psychology of Women Quarterly 1996; 20: 147-69.

23. CÁCERES CF. Assessing young people's non-consensual sexual experiences: Lessons from Perú. Pp 127138 en Jejeebhoy, Shah \& Thapa (eds.), Sex Without
Consent: Young People in Developing Countries. London: Zed Books; 2005.

24. ULman SE. A critical review of field studies on the link of alcohol and adult sexual assault in women. Aggression and Violent Behavior 2003; 8: 471-86.

25. RICKERT VI, WiEMANN CM. Date rape among adolescents and young adults. J Pediatr Adolesc Gynecolo 1998; 11: 167-75.

26. Searle J. The Construction of Social Reality, New York: The Free Press; Harmondsworth, Middlesex: Penguin Books; 1995.

27. Beitchman JH, Zucker KJ, Hood JE, Dacosta GA, AKMAN D, CASSAVIA E. A review of the long-term effects of child sexual abuse. Child Abuse and Neglect 1992; 16: 101-18.

28. Messman TL, Long PJ. Child sexual abuse and its relationship to revictimization in adult women: A review. Clin Psychol Rev 1996; 16: 397-420.

29. Lewis SF, FrEMouw W. Dating violence: A critical review of the literature. Clin Psychol Rev 2001; 21: 105-27.

30. Koss MP, Goodman LA, Browne A, Fitzgerald LF, Keita GP, Russo NF. No Safe Heaven: Male Violence Against Women at Home, at Work, and in the Community. Washington, DC: American Psychological Association; 1994. 